Accretion Phenomena and Related Outflows, IAU Colloquium 163

ASP Conference Series, Vol. 121, 1997

D.T. Wickramasinghe, L. Ferrario, and G.V. Bicknell, eds.

\title{
Accretion Discs around Black Holes
}

\author{
Rainer Wehrse \\ Institut f. Theoretische Astrophysik, Universität Heidelberg, \\ Tiergartenstr. 15, D-69121 Heidelberg, Germany \\ Interdisziplinäres Zentrum für Wissenschaftliches Rechnen, Universität \\ Heidelberg, Im Neuenheimer Feld 368, D-69120 Heidelberg, Germany
}

\begin{abstract}
.
The structure of accretion discs around (super-) massive black holes is discussed in this contribution with special emphasis on the radiation fields. These are of crucial importance for the understanding of these objects since photons control in most cases not only the temperature distributions but also the pressures and shapes. Recent progress in the modelling of photon fields now provides the means for a much improved understanding of the consequences of the multidimensional structure of the discs as well as of the effects of the strong space time curvature and of the high velocities involved. However, the simultaneous inclusion of the NLTE level populations and of many spectral lines is still a major problem. It is also demonstrated that special and general relativity effects strongly distort the apparent brightness distributions and spectra such accretion discs so that a solution of the inverse problem will be very difficult.
\end{abstract}

\section{Introduction}

Observational evidence is mounting that black holes really exist in the universe and that they accrete matter from their surroundings. In fact, they seem to be quite ubiquitous and very spectacular so that the proper modelling is highly desirable. Unfortunately, it is also immediately evident that the full modelling of such object implies the study of a plasma far away from LTE (perhaps even the NLTE requirements hold no longer) under the influence of gravity as well as self-produced magnetic and radiation fields in a curved space time. A corresponding consistent treatment is presently (and probably for the next decade at least) completely out of reach. Since hydrodynamic and magneto- hydrodynamic aspects are addressed in several contributions in these proceedings and have been reviewed elsewhere, this article mainly deals with the corresponding radiation fields.

Simple estimates on the basis of height integrated accretion discs of ShakuraSunyaev type show (Frank, King, Raine 1992) show that in accretion discs around black holes the radiation fields play an important role for the structure: not only do they influence largely the thermal structure but in particular they give rise to the radiative force in the vertical direction which may closely 
compensate the vertical component of the gravity and therefore essentially determine the shape of the disc.

Unfortunately, we are only now starting to learn how to model such radiation fields. The reason for this situation is the dependence of the basic quantity of radiometry, the specific intensity, on seven parameters ( 3 spatial and 2 angular coordinates, frequency and time) and the intricate couplings by the radiative transfer equation. Useful analytical solutions are therefore not available. In numerical approaches additional difficulties arise from the fact that black hole accretion discs have rather strong temperature and pressure gradients in regions of interest (e.g. the photosphere) so that tensor-product grids can hardly be employed and from the fact that usually scattering is much stronger than absorption.

\section{Radiative Transfer in Black Hole Accretion Discs}

\subsection{D Radiation Fields}

If bulk motions, general relativity and polarisation effects are neglected the specific intensity $I$ in an accretion disc is given by the radiative transfer equation

$$
\frac{1}{c} \frac{\partial I(t, \nu, \mathbf{x}, \mathbf{n}}{\partial t}+\nabla \cdot I(t, \nu, \mathbf{x}, \mathbf{n})=-\chi(t, \nu, \mathbf{x})(I(t, \nu, \mathbf{x}, \mathbf{n})-S(t, \nu, \mathbf{x}))
$$

( $c=$ speed of light, $t=$ time, $\nu=$ frequency, $\mathbf{x}=$ vector of spatial coordinates, $\mathbf{n}=$ unit direction vector, $S=$ source function).

Fortunately, except in regions of strong radial motion the hydrodynamic timescales are much longer than the time scales for the light propagation and for the establishment of (statistical) equilibrium level populations and therefore the explicit time dependence in Eq. 1 can usually be neglected and the evolution of the radiation field can be approximated by a sequence of snapshots each reflecting a corresponding hydrodynamic state. However, due to the spatial couplings in the transport operator and the angle (and possibly wavelength) coupling in the source function the solution of even this highly simplified equation is very demanding, e.g. usually, more than $10^{8}$ linear equations have to be solved simultaneously. The use of adaptive unstructured grids and the employment of sophisticated solvers for the linear equations is therefore of utmost importance (cf. contribution of Kanschat in this volume). But even if such elaborate methods are used the cpu and memory requirements are enormous.

\subsection{Effects of Velocity Fields}

In order to take the effects of the orbital motion into account (cf.Mihalas and Weibel-Mihalas, 1984; Papkalla, 1994) the transfer equation is usually Lorentz transformed and the "resulting transfer equation in the comoving frame" is solved e.g. by means of the method of short characteristics. However, this turns out be be quite difficult since in discs both positive and negative relative velocities are found and therefore one has to deal with a boundary value problem for the frequencies, also. It turns out (Baschek et al., 1996) that -at least in cases of given source functions- it is advantageous (i) to rewrite the solution of the corresponding $1 \mathrm{D}$ transfer equation in terms of the relativistic invariant 


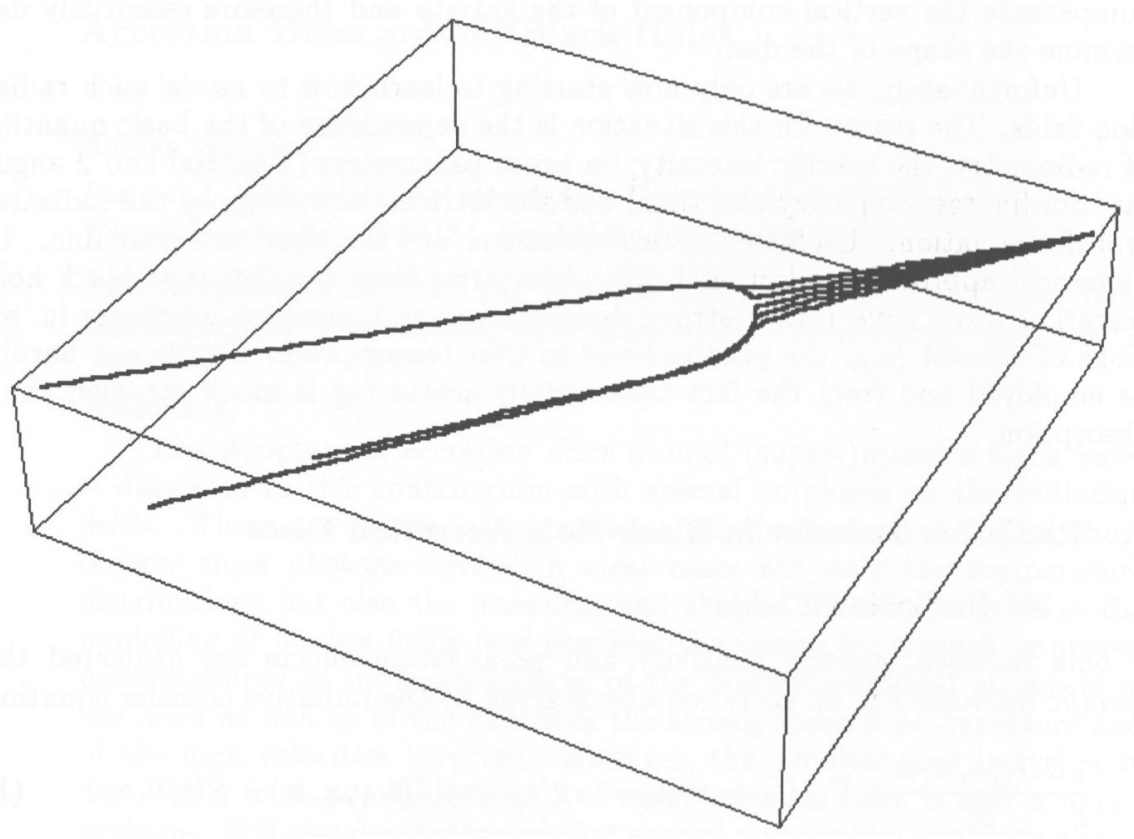

Figure 1. Photon paths due to the orbital motion of the matter as seen by a distant observer that does not move with respect to the centre. Only special relativity effects are considered.

specific intensity, (ii) to make a point transformations to represent the resulting line integral in the correct frame and (iii) to perform the appropriate Lorentz transformation.

In the case of black hole accretion discs the full Lorentz transformation has to be applied as aberration/advection effects (Fig. 1) and transversal Doppler effects are quite strong. They should show up in particular in lines due the fast variation of the absorption coefficient with frequency and direction (for the latter see Fig. 2).

\subsection{Effects of Space-Time Curvature}

The importance of space-time curvature for the propagation of radiation from black hole accretion discs has been stressed already by Cunningham (1975; see also Laor and Netzer, 1989) who provided transfer functions that connect the intensities emitted by an infinitely extended flat plate around a black hole with those observed by a distant observer. However, this is an inadequate for accretion discs (cf. Viergutz, 1993) since (i) it does not treat the transfer in the disc itself, (ii) it in hibits rays from the "lower side" of the disc to reach the observer, and (iii) it does not allow indirect images to occur. Although, to my knowledge, the first problem has not yet been solved the fact that space-time curvature is small over a photon mean free path seems to indicate that the corresponding effects are not 


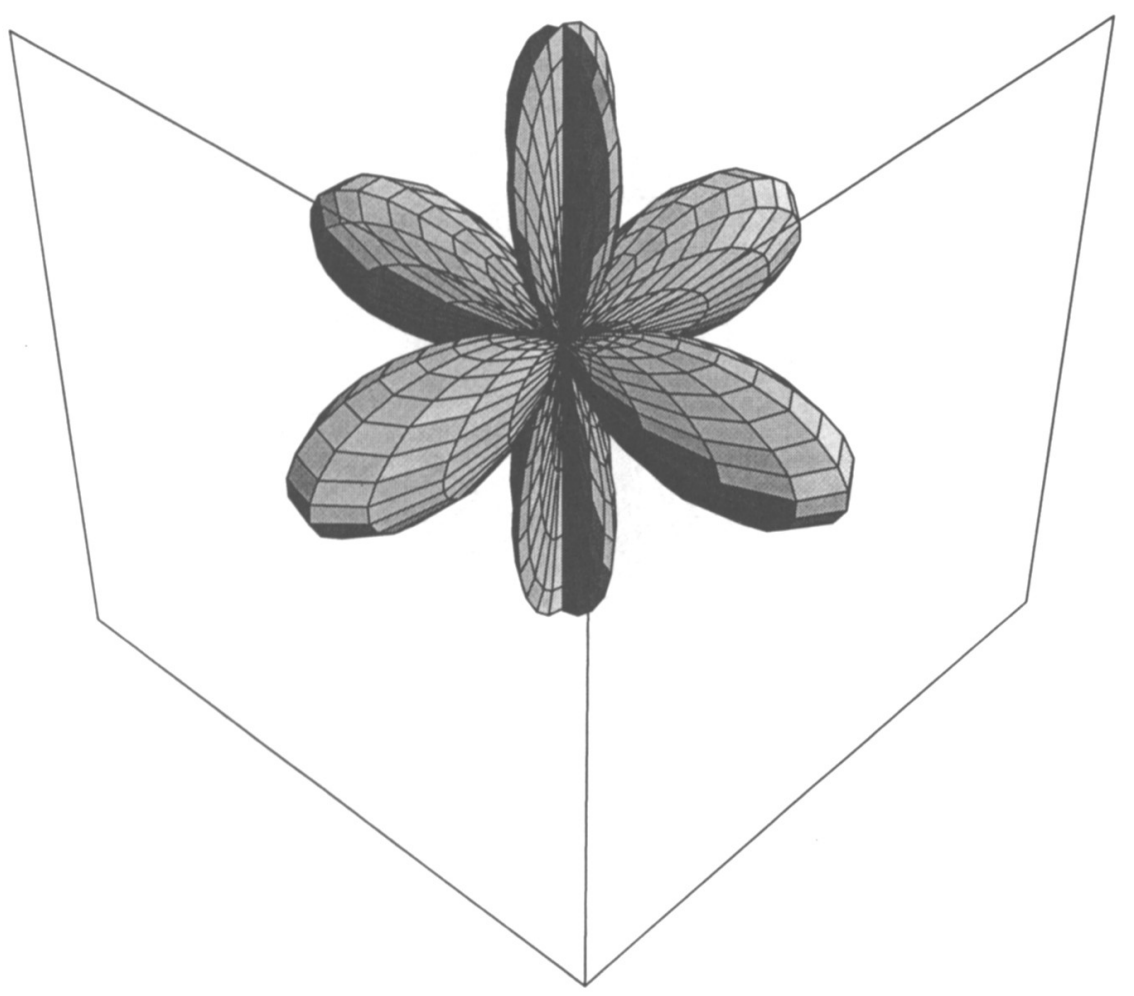

Figure 2. Typical angle distribution of the line extinction coefficient as "seen" by a photon at line centre. 


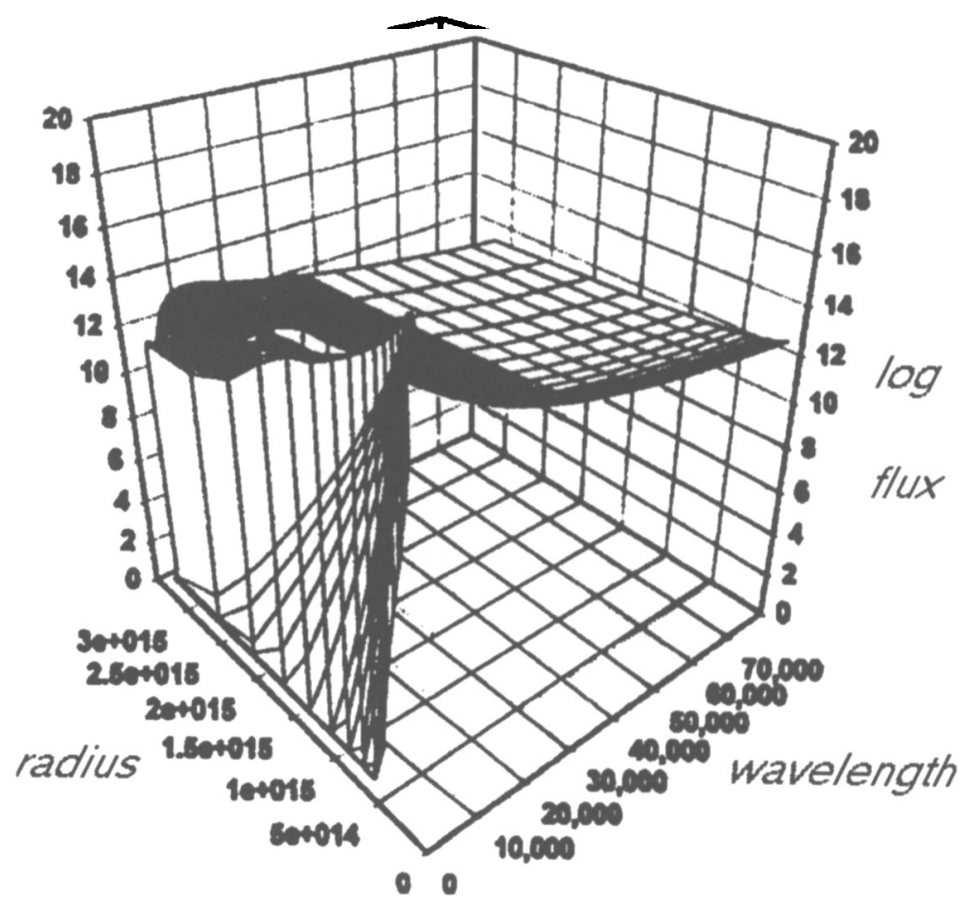

Figure 3. Example of a flux distribution as emitted by the surface of an accretion disc surrounding a maximally rotating supermassive black hole as measured by an observer corotating with the disc matter. From Kindl, 1994. 
Figure 4. Distortion of a homogeneous flat disc by a maximally rotating black hole, see text. From Kindl, 1994.

Figure 5. Grey scale representation of the spatially resolved brightness of the accretion disc of Fig. 3 as seen by a distant observer at $880 \AA$. The inclination angle is 30 degrees. Zero relative motion of the observer and the centre of the configuration is assumed. From Kindl 1994, see also Quien et al. 1995. 


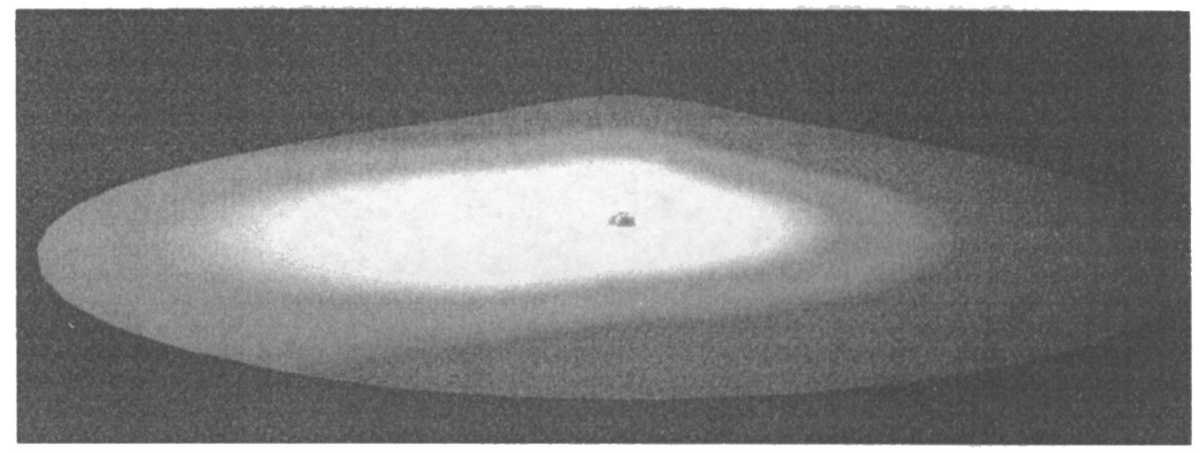

Figure 6. Same as Fig. 5 but for observer wavelength $\lambda=3600 \AA$.

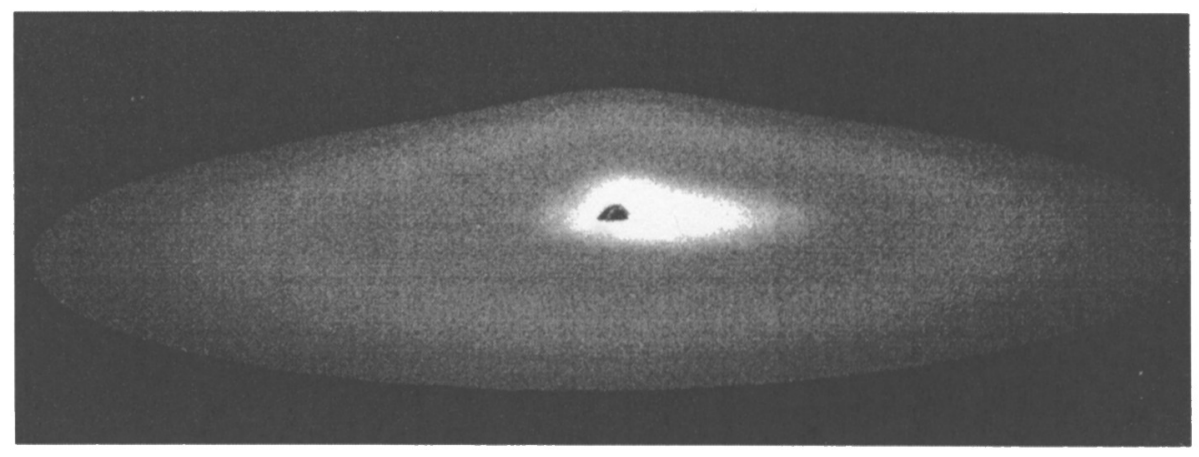

Figure 7. Same as Fig. 5 but for observer wavelength $\lambda=10^{4} \AA$. 


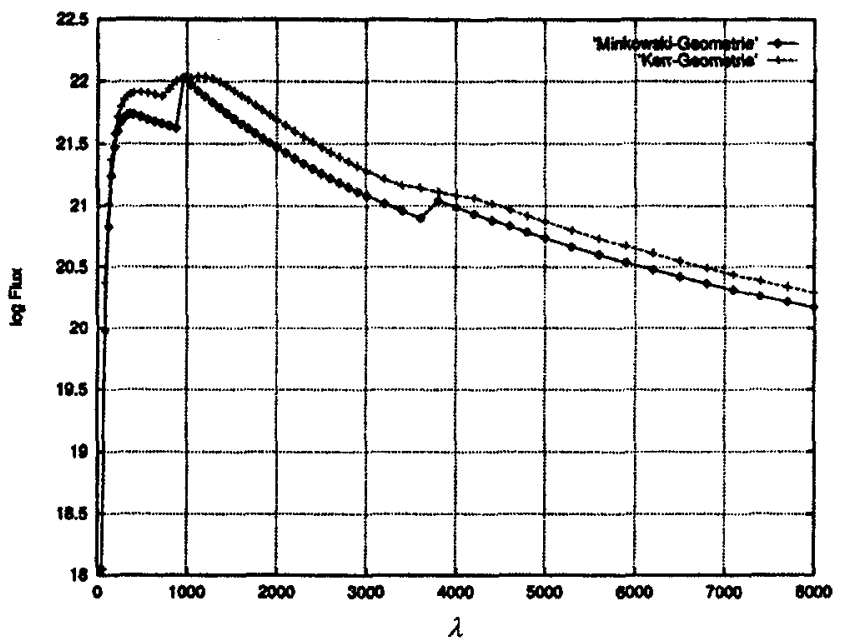

Figure 8. Consequences of orbital motion and space-time curvature on the total disc spectrum as seen by a distant observer at rest with respect to the centre of the configuration for Minkowski and Kerr geomtry. Note in particular the essential disappearance of the Lyman edge in the Kerr case. 


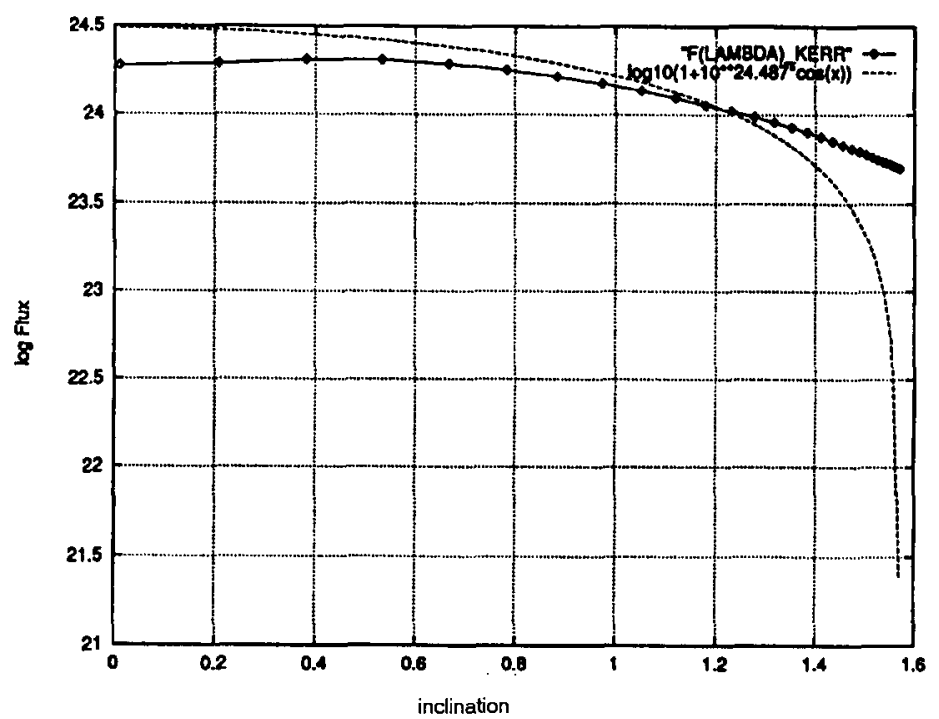

Figure 9. Variation with inclination angle of the total disc flux as measured by a distant observer at $3600 \AA$ for Minkowski and Kerr geometry. The turn-up for large inclination angles in Kerr geometry results from the appearance of the lower central regions that are classically hidden.

very large. The problems (ii) and (iii) have been overcome by the very elegant semi-analytical algorithm of Viergutz (1993) that reduces the connection of the emitter and receiver directions to the solution of two coupled transcendental equations. In most cases this approach also allows for the effective determination of surfaces that are not seen by an observer. The problems can also be solved by a generalisation of the classical ray tracing technique (Quien, 1994; see Kindl, 1994; Speith et al., 1995, Marck, 1996). Such an approach is highly flexible but it requires an enormous amount of computer time and -if indirect images are to be considered- a very careful consideration of the integration step width in the vicinity of turning points.

\subsection{Spatially Resolved Brightness Distributions}

The consequences of space-time curvature and the frequency shifts due to the gravitational and velocity fields show up particularly in the monochromatic brightness distribution of black hole discs as they would appear to a distant observer. In order to demonstrate the effects let us assume (i) that black hole is rotating with maximal speed (and therefore a Kerr metric has to be considered) (ii) that the disc intrinsically has axial symmetry and (iii) that the radial and wavelength dependence of the local surface emission is either negligible or that of 
a fully iterated non-grey model (Fig. 3) which has been calculated essentially according to the prescription of Shaviv and Wehrse (1991, see also Störzer, 1993). The main modification due to the metric as seen by a distant observer is the upbending of the upper back part of the disc (see Figs. $4-7$ ) and the simultaneous down-bending of lower back part which causes that for not too small inclination angles light from both sides of the disc reaches the observer at the same time in contrast to the situation in Minkowski space. Since this applies in particular to the hot central region fluxes at short wavelengths are enhanced. The second effect of the metric is the destruction of the right/left symmetry due to black hole rotation (to be distinguished from the un-symmetries caused by boosting and frequency shifts, see below). In addition (but not seen in the figures), the bending of the light rays increases the surface heating due to illumination of flared parts of the disc and cause the formation of indirect images (Viergutz, 1994; Quien, Wehrse, Kindl, 1995) by photons that go completely around the black hole at least once.

The final effect of general relativity is the gravitational redshift. It is superimposed by the Doppler shift and boosting due to the orbital motion in the disc. The latter induce asymmetries whenever there is a flux gradient $\partial F / \partial \lambda \neq 0$. As is clearly seen from Figs. 5 to 7 it implies that the apparent brightness centre is wavelength dependent and that in cases where the comoving frame wavelengths comprise spectral edges particularly strong shifts of the brightness centre from the geometrical centre occur.

\section{Discussion and Conclusions}

The modifications in the radiation field due to the metric are so strong that they also show up clearly in the spatially integrated spectra (Fig. 8). Most remarkable is the smearing out of sharp spectral features, as e.g. the Lyman jump. Whereas this property improves the agreement with observations (cf. Ulrich, 1989) it unfortunately lowers considerably the information content of the spectra (Hafner and Wehrse, 1993) and therefore makes detailed spectral diagnostics very difficult. As a consequence, for the solution of the inverse problem it is necessary to use a frequency band as wide as possible and to obtain as many parameters from non-spectroscopic information, as e.g. time variability. Another "global consequence" of the bending of light rays is an increase in the mutual illumination of different parts of the disc resulting partially in a reflection by scattering electrons and partially by the heating of the uppermost disc layers.

The variation of the observed flux of the whole disc with the inclination angle may also be affected considerably by the metric (Fig. 9). In particular, the emission of black hole accretion discs is much more isotropic than for corresponding discs in a Minkowski space (facilitating -among others- the empirical determination of the total luminosity). Evidently, the flux is increased for the black hole case if simultaneously the upper and lower side of the central region are visible.

In conclusion, recent progress in the modeling of radiation fields ( $3 \mathrm{D}$ configurations, velocity fields, space-time metric) has added many interesting features to our knowledge of accretion discs around black holes. On the other hand, the collective effects of many spectral lines and the role of NLTE may also be very 
significant but can presently not be estimated; corresponding investigations have urgently to be started.

Acknowledgments. Financial support by the Deutsche Forschungsgemeinschaft (SFB 359/C2 and a travel grant) is acknowledged.

\section{References}

Baschek, B., Efimov, G.V., v. Waldenfels, W., Wehrse, R. 1996 A\&Ain press Cunningham, C.T. 1975, ApJ 202, 788

Frank, J., King, A., Raine, D. 1992, Accretion Power in Astrophysics, 2nd. ed., Cambridge Astrophys. Ser. 21

Hafner, M., Wehrse, R. 1994, A\&A 282, 874

Kindl, C. 1994, diploma thesis, Heidelberg University

Laor, A., Netzer, H. 1989, MNRAS 238, 897

Marck, J.-A. 1996, private communication

Mihalas, D., Weibel-Mihalas, B. 1984, Foundations of Radiation Hydrodynamics, Oxford Univ. Press

Papkalla, R. 1995, A\&A 295, 551

Quien, N., Wehrse, R., Kindl, C. 1995, Spektrum d. Wissenschaften, May 1995, p. 56

Shaviv, G., Wehrse, R. 1991, A\&A 251, 117

Speith, R., Riffert, H., Ruder, H. 1995, Comp. Phys. Comm. 88, 109

Störzer, H. 1993, A\&A 271, 25

Ulrich, M.-H. 1989, in: Theory of Accretion Disks, Meyer, F., et al., eds., Nato ASI Ser. C, vol 290, p. 3

Viergutz, S. 1993, A\&A 272, 355

\section{Discussion}

R. Soria: Would you please comment on the problem of stability of the disc? The disc tends to evaporate in your models, is it really a physical effect and what does it depend on?

R. Wehrse: Evaporation can occur in our models as a consequence of an imbalance of heating and cooling in the very high disk layers. Unfortunately, at present we cannot model properly the cooling by lines (extremely complicated radiative transfer, NLTE, frequency redistribution, ...) 